\title{
FACTORS AFFECTING LASER-TRIM STABILITY OF THICK FILM RESISTORS
}

\author{
R. E. COTE and R. C. HEADLEY \\ E. I. du Pont de Nemours \& Co., Inc., Photo Products Department, \\ Electronic Materials Division, Niagara Falls, New York, U.S.A. \\ J. T. HERMAN \\ E. I. du Pont de Nemours \& Co. Inc., \\ Engineering Department, Wilmington, Delaware, U.S.A. \\ A. HOWE \\ Teradyne Incorporated, Chicago, Illinois, U.S.A.
}

(Received June 14, 1977)

\begin{abstract}
Various factors affecting precision of trim and resistor stability are considered. The influence of machine operating parameters (beam power, pulse frequency and trim speed) on resistor performance are examined and quantified through statistically designed experiments for a $Q$-switched YAG laser system. Laser kerf quality is examined by scanning electron microscopy and related to kerf isolation resistance measurements. A relatively simple, production oriented, quality control test is proposed for rapid determination of kerf electrical stability. In addition, the effect of cut design and extent of trim on precision and stability are discussed.
\end{abstract}

\section{MACHINE RELATED PARAMETERS}

A consideration of factors that affect the precision of trim and stability of laser trimmed thick film resistors should begin with an examination of some of the aspects of the laser system itself. Specifically, one should concern himself with those parameters which are generally adjusted in the equipment set-up and then assumed to remain constant through the laser trimming process. These consist of beam spot size and sharpness of focus, average beam power, pulse frequency, probe stability, bridge tracking response and settling time. Some of these factors contribute to the quality of the kerf. Others influence the measurement system and can lead to false interpretation of data.

Peak power, not average power, removes material. However, peak power is extremely difficult to measure and average power is what one normally measures. One of the factors which affects peak power in a most critical way is spot size. When a laser is operated in TEM ${ }_{\text {oo }}$ mode (that is, a single spot output from the laser) the distribution of the energy output is nearly Gaussian, as shown in Figure 1A.
The beam is then passed through a series of focusing lenses shown diagrammatically in Figure 1B as a single lens. Laser energy in this mode does not focus to an infinitely small spot as does dispersive radiation. At the point of focus, the beam exhibits a minimum waist diameter determined by the lens' focal properties. The beam intensity is then sufficient to vaporize material and cut through the resistor material.

It is important to note that peak power varies inversely with the square of beam diameter. If a minimum power required to remove material is defined, we see in Figure 1C that a laser operated in poor focus will direct energy of lower intensity into the walls of the laser kerf. This will cause damage due to thermal shock and will not remove material cleanly. In extreme cases, microcracks and fissures may be produced.

The use of apertures can improve beam focus, particularly with multimode (donut or multispot beam) propagation, by taking a "prime cut" out of the energy distribution. Figure 2 shows this diagrammatically. The aperture blocks most of the divergent radiation of the laser energy output which 


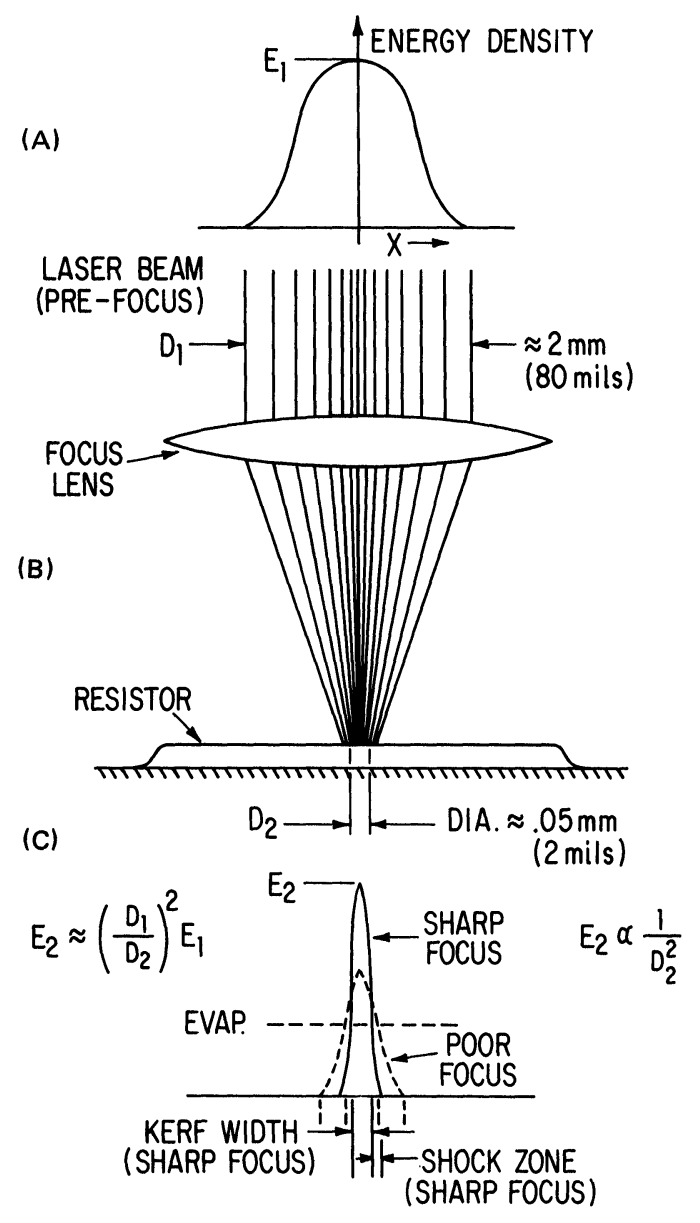

FIGURE 1 Focus.

would otherwise degrade the focus of the beam.

Average beam power is an easily measured machine parameter. It is directly adjustable by panel control of pump lamp current and programmable with most modern computerized systems. When it is varied with other parameters fixed, a relationship between average beam power and post trim resistor stability may be observed for most thick film resistor materials. Figure 3 shows this relationship in generalized form. An optimum level of resistor stability, $\Delta R_{0}=1$, is obtained over a limited range of beam power. Extensive inspection of trimmed resistors has shown that below or above this limited range, kerf quality deteriorates in either of two principal ways. These are; incomplete removal of material (low power) or wider peripheral zones of disturbed material bordering the kerf (high power). In the latter case, the extent of damage is often evidenced by microcracks extending into functional

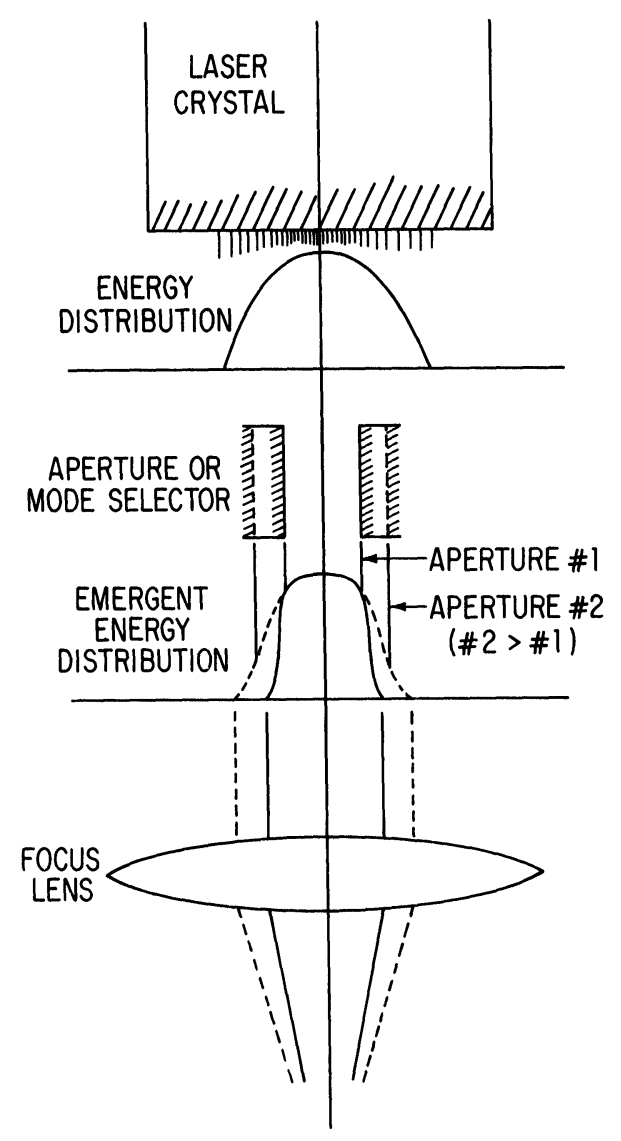

FIGURE 2 Spot size.

areas of the trimmed resistor. Two other effects to be seen with high beam power are wider kerfs and less well-defined kerf walls.

Ideally, trimming should not result in further resistance change after completion of trim. Laser energy flow into resistor material outside the trim path, or into the substrate should, therefore, be minimized to maintain high integrity of the trimmed configuration. That is, a maximum of laser energy should be absorbed in the process of resistor material vaporization. This requires pulsed energy of high peak power and pulses of short duration. These conditions are not independent of pulse frequency or $Q$-rate, and stability problems do occur where high pulse frequencies have been used to facilitate high trim speeds. Figure 4 provides a basis as to why this may happen.

The relationship between peak power and pulse duration as the $Q$-rate is varied was determined experimentally using photocell-oscillographic techniques. Peak power is shown on a relative scale 


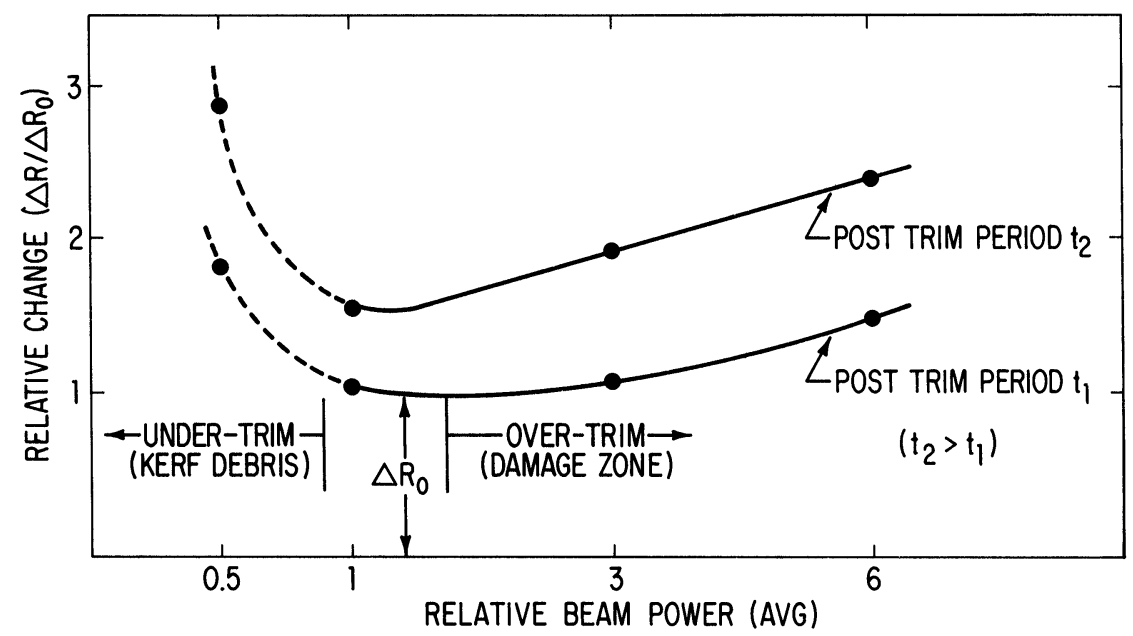

FIGURE 3 Post trim stability vs. avg. beam power.

which corresponds to photocell output (volts). Pulse duration corresponds to the time interval between the pulse half-power points. Figure 4 shows a noticeable drop-off in peak power as $Q$-rate increases. At the same time, pulse duration increases. The result is less pulse energy of sufficient magnitude to achieve rapid vaporization and more energy flowing into the resistor body and substrate. The consequences of very high $Q$-rates are visible as pronounced reflow along kerf walls accompanied by stress induced cracking in the reflowed areas. Inadequate kerf formation also occurs. This study is not complete in that the influence of input pump lamp power has been investigated only up to the level where $\mathrm{TEM}_{\mathrm{oo}}$ mode propagation predominates.

Any measurement system requires a finite time to achieve balance and measure resistors. During the trimming operation, resistance values increase by discrete steps the magnitude and frequency of which are determined by the laser pulse conditions, cut

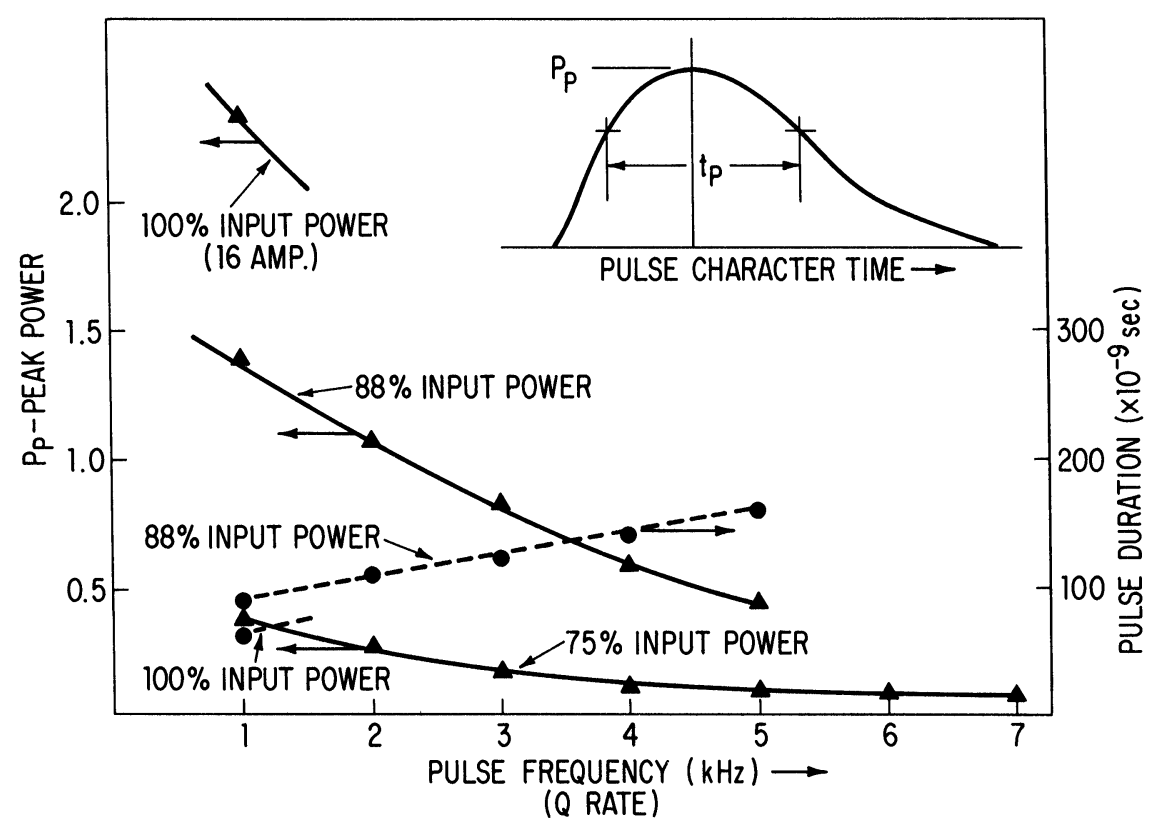

FIGURE 4 Pulse frequency. 
mode, resistor size and extent of trim. The bridge tracks the resistance values between laser pulses. If the magnitude and frequency of resistance change does not allow the bridge enough time to reach balance, an accumulating error in measurement will occur as trimming proceeds. The result is overshoot. This is shown in Figure 5 where overshoot is shown to be equal to the measurement lag.

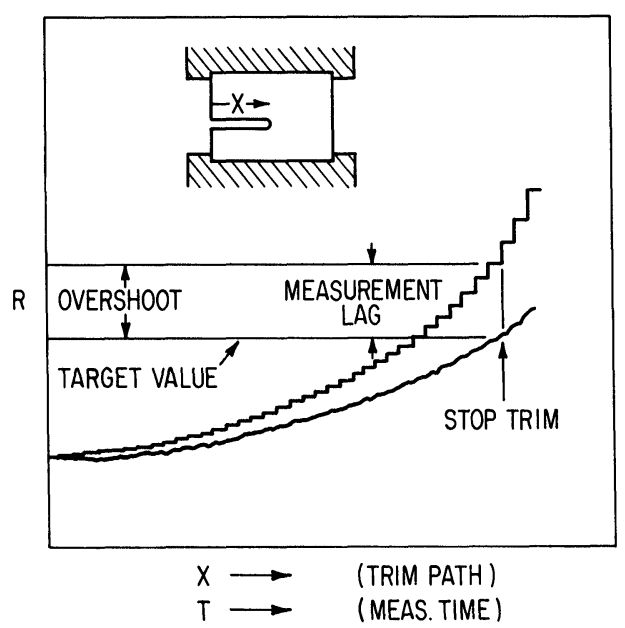

FIGURE 5 Bridge tracking response.

The problem becomes more severe when high value resistors, in excess of 1 megohm, are trimmed. Measurement lag and overshoot can exceed $1 \%$ and predictability can also be lost. In some very high resistance values, the resistor can be trimmed completely open unless sufficiently large cut-offs or adequate delays are programmed into the measurement or provisions are made at the probe ring to speed the measurement. To overcome this problem, it may also be necessary to slow the trimming speed. Tracking response effects can also be minimized by the use of multiple cuts where the final cut requires a maximum $2-3 \%$ change. Other factors that influence overshoot are temperature coefficient of resistance and intrinsic stability characteristics of the resistor being trimmed.

In addition to the bridge tracking response, another factor which affects the accuracy of the measurements is bridge settling time. This factor is present whether or not the resistor is being trimmed. The time interval required to obtain a given measurement accuracy increases as the magnitude of the resistance value becomes larger. A variety of measurement modes are available with modern laser systems which permit rapid, low precision measurements, or slower, more precise measurements.

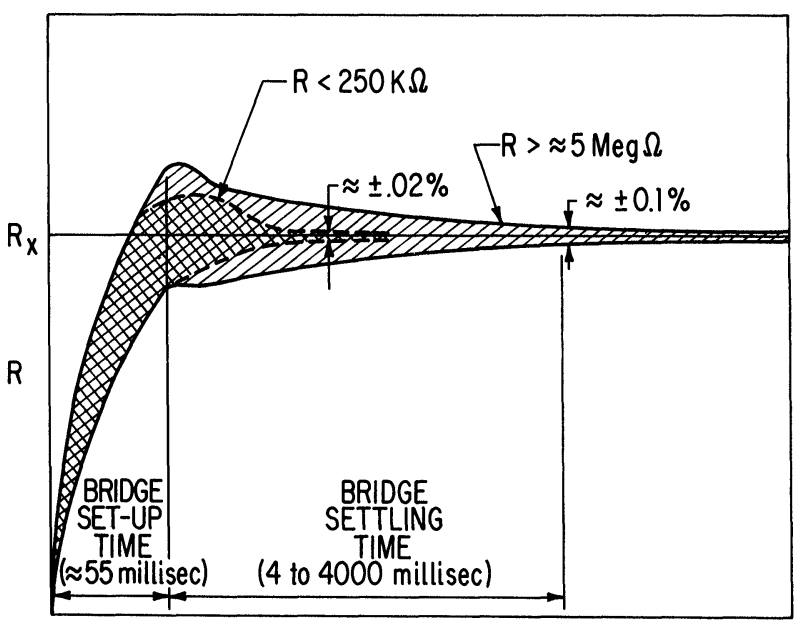

TIME $\rightarrow$

*ESI Mod. 25 YAG System. Auto Zero Mode.

FIGURE 6 Bridge settling time.

These "modes," as well as settling time, are usually programmable, and care should be taken to select the best combination where accurate measurements are required. In Figure 6, the influence of time on measurement accuracy is illustrated by the shaded areas. The figure is intended to provide an indication of the magnitudes involved, and the numbers should not be taken literally.

A final point worth mentioning involves the probe station (Figure 7). Measurement probes are often

(A)

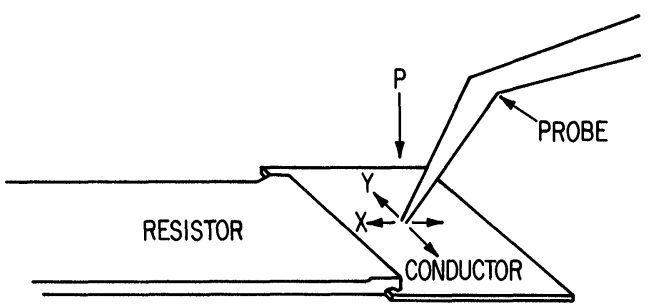

(B)

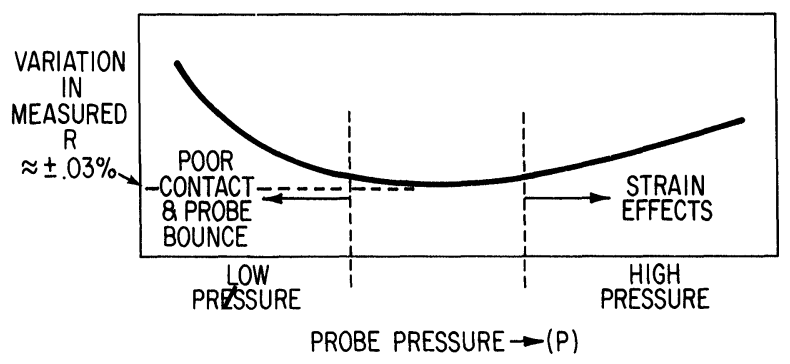

FIGURE 7 Probing stability. 
TABLE I

Experimental design

\begin{tabular}{|c|c|c|c|c|}
\hline Run No. & Sample Nos. & $\begin{array}{l}\text { Pulse Frequency } \\
(\mathrm{kHz})\end{array}$ & $\begin{array}{l}\text { Trim Speed } \\
\text { (in./sec.) }\end{array}$ & $\begin{array}{l}\text { Average Beam } \\
\text { Power } \\
\text { (Watts) }\end{array}$ \\
\hline 1 & $1-10$ & 3 & 1 & 1.0 \\
\hline 2 & $11-20$ & 5 & 0.1 & 0.5 \\
\hline 3 & $21-30$ & 3 & 1 & 0.5 \\
\hline 4 & $31-40$ & 1 & 2 & 0.5 \\
\hline 5 & $41-50$ & 1 & 2 & 1.5 \\
\hline 6 & $51-60$ & 5 & 2 & 0.5 \\
\hline 7 & $61-70$ & 5 & 0.1 & 1.5 \\
\hline 8 & $71-80$ & 1 & 0.1 & 0.5 \\
\hline 9 & $81-90$ & 1 & 2 & 1.5 \\
\hline 10 & $91-100$ & 1 & 1 & 1.0 \\
\hline 11 & $101-110$ & 3 & 1 & 1.0 \\
\hline 12 & $111-120$ & 3 & 2 & 1.0 \\
\hline 13 & $121-130$ & 5 & 0.1 & 1.5 \\
\hline 14 & $131-140$ & 5 & 2 & 0.5 \\
\hline 15 & $141-150$ & 1 & 0.1 & 0.5 \\
\hline 16 & $151-160$ & 5 & 2 & 1.5 \\
\hline 17 & $161-170$ & 5 & 1 & 1.0 \\
\hline 18 & $171-180$ & 3 & 0.1 & 1.0 \\
\hline 19 & $181-190$ & 1 & 0.1 & 1.5 \\
\hline 20 & $191-200$ & 3 & 1 & 1.5 \\
\hline 21 & $201-210$ & 3 & 1 & 1.0 \\
\hline
\end{tabular}

fragile and subject to vibration and rapid wear. Probe pressure should be of concern for a particular probe style. Long, cantilevered probes are prone to bounce, which can lead to erratic results if the pressure is too low or an adequate delay between probe actuation and measurement time is not maintained. This is most serious in measurements of low resistance values where a significant contribution can result from high contact resistance. Also, stability of the probe placement can be critical to obtaining reproducible results. All thick film resistors exhibit strain effects to a greater or lesser degree. In this respect, excessive, unstable probe pressure, uneven substrates, and poor or dirty nesting can compromise the measured results.

\section{EFFECT OF FREQUENCY, SPEED AND AVERAGE POWER}

An experiment was statistically designed to study the relative effects of trim speed, $Q$-rate and power on precision of trim and resistor stability. The experimental design took the form of a face-centered cube illustrated by Figures $8-10$. The test matrix included the eight corner points of the cube, the six face points, the center point repeated three separate times and four replicates of the corner points. The experimental design is shown in Table I.

The machine parameters were varied over the following ranges: average power, 0.5 to 1.5 watts; cutting speed, 2.5 to $50 \mathrm{~mm} / \mathrm{sec}$. (0.1 to 2.0 inches/ sec.); $Q$-rate, $1 \mathrm{kHz}$ to $5 \mathrm{kHz}$. Fired films with sheet resistivities of $100 \Omega / \square, 10 \mathrm{k} \Omega / \square$ and $1 \mathrm{M} \Omega / \square$ were studied, with each result the average of data points from measurements of 40 resistors. All resistors were $1 \mathrm{~mm}^{2}$ (0.040 in. $\times 0.040$ in.) trimmed with a single plunge cut. The spread of resistance values of the as-fired resistors was approximately $\pm 15 \%$ around a mean value, and the latter was assumed to be $33 \%$ below a hypothetical target value. Thus, on the average, trimming raised the value by a factor of 1.5 with some resistors being "trimmed up" by a factor of 2.

All of the laser trimming and measurement was carried out with a Teradyne Model W311, $Q$-switched, YAG laser system. Initial deviation from target (precision of trim) and 24-hour and 100-hour percentage resistance drift (referenced to the initial measurement) were measured.

The results of the experiment are shown in Figures 8-10. It can be seen that speed of trim has 
Initial \% Deviation From Target (Precision of Trim)

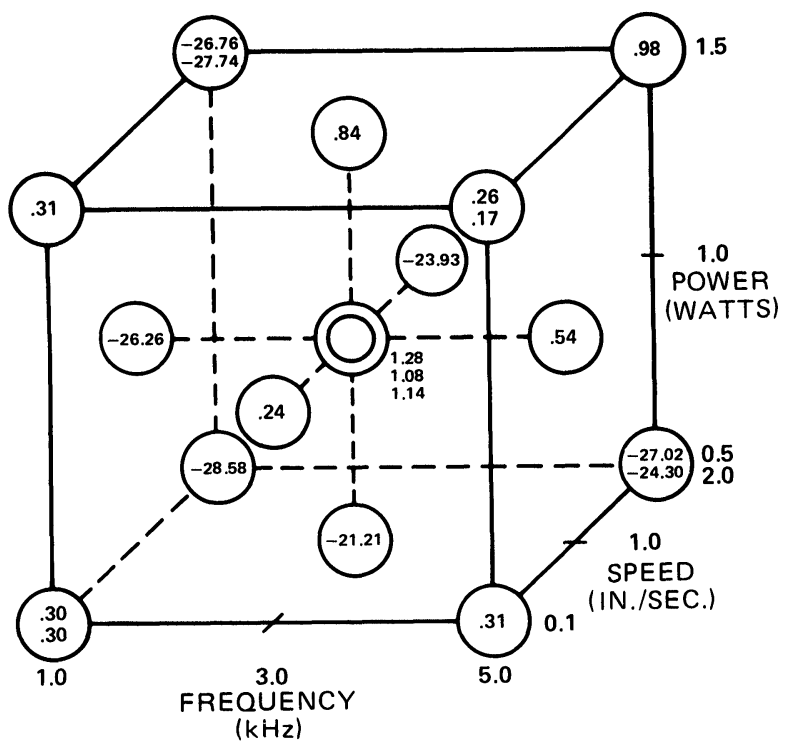

\section{Hour Drift $(\% \Delta R)$}

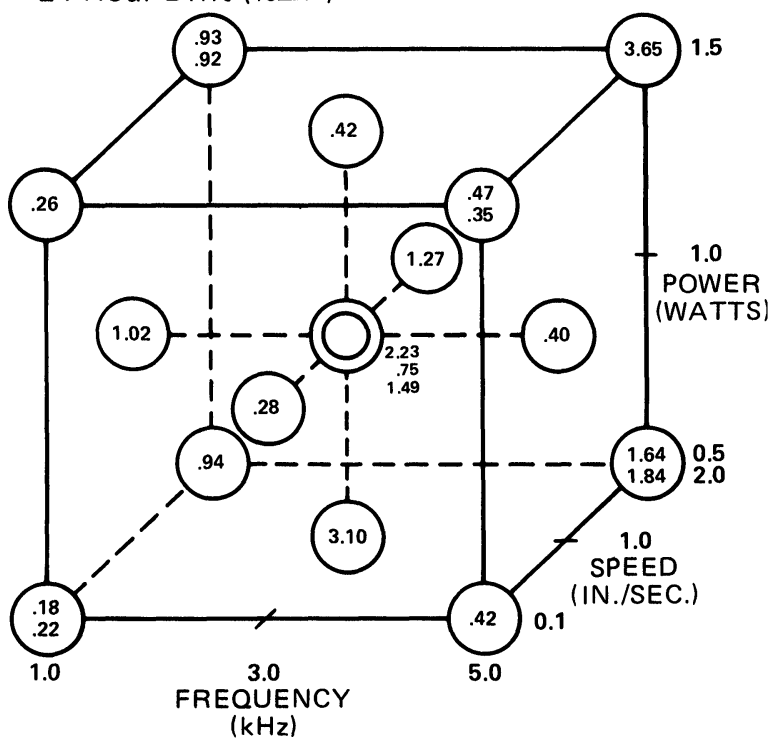

100 Hour Drift $(\% \Delta R)$

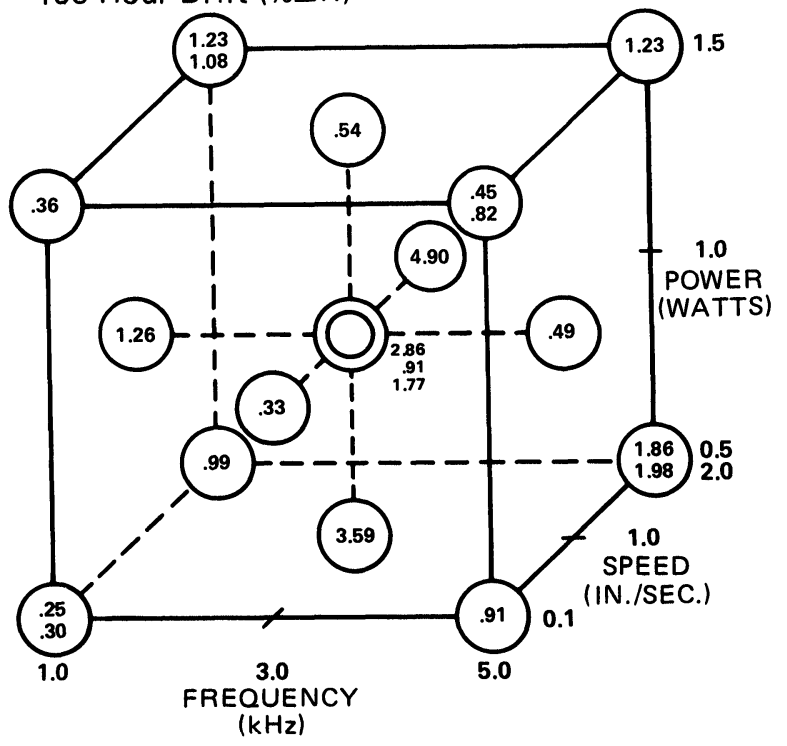

FIGURE 8 Laser trimming of "Birox" $1421(100 \Omega / \mathrm{sq}$.$) Initial \%$ deviation from target (precision of trim).

the largest effect on both precision and drift. This was borne out by a regression analysis which showed speed to be the greatest single contributor but also showed some interdependency of the three variables.

The experiment yielded "volumes" within the experimental cube where combinations of speed, frequency and power produce equivalent results within experimental error. It also indicates that different conditions may be required to achieve satisfactory results with low ohm materials compared to high ohm resistor compositions.

Figure 10 presents data for the $1 \mathrm{M} \Omega / \square$ material. The first cube refers to precision of trim. It is apparen that the trim conditions at the left end, front edge of the cube - the lowest speed and frequency somewhat independent of power - yield the smallest 
Initial \% Deviation From Target (Precision of Trim)

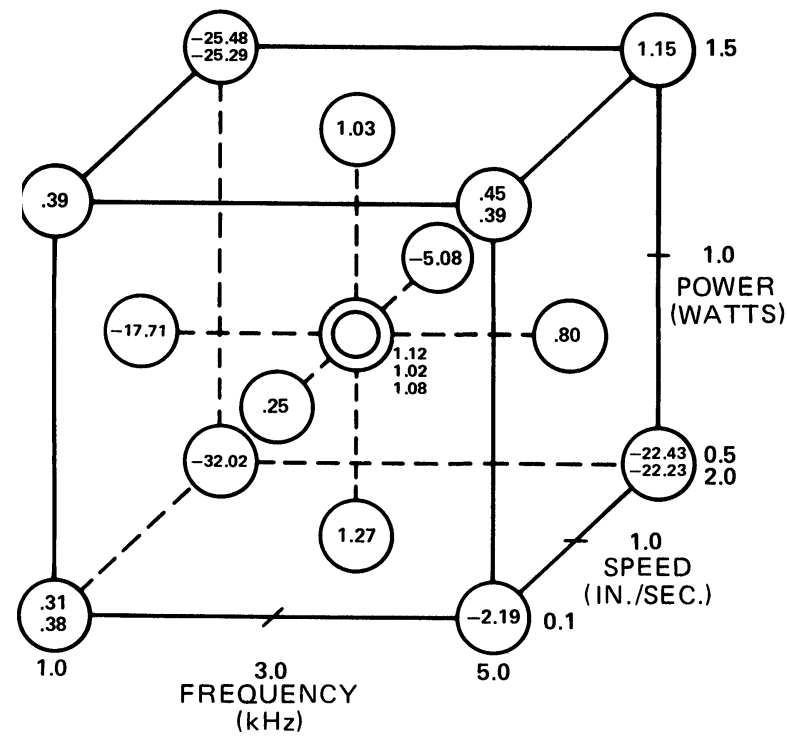

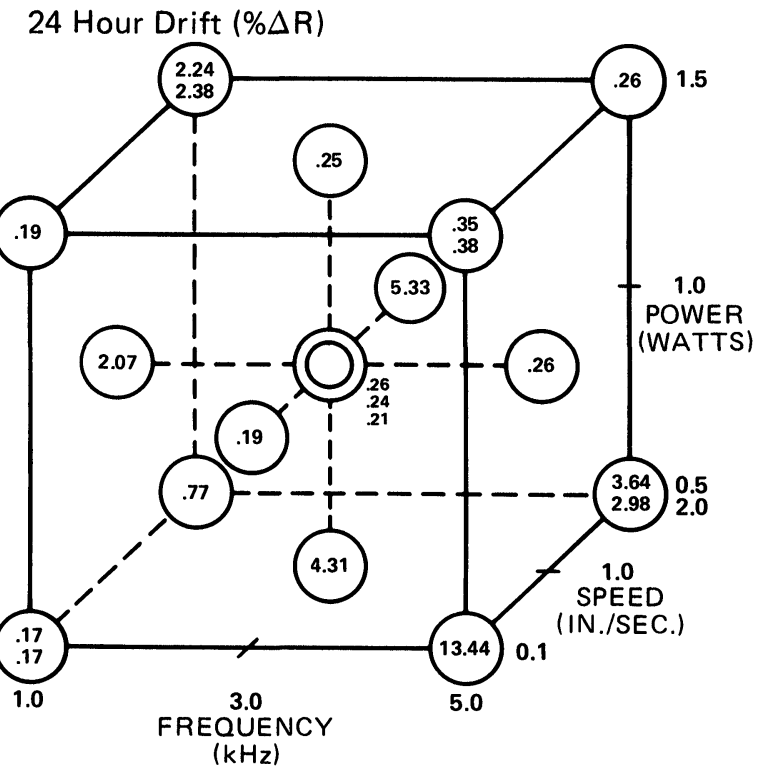

100 Hour Drift $(\% \Delta R)$

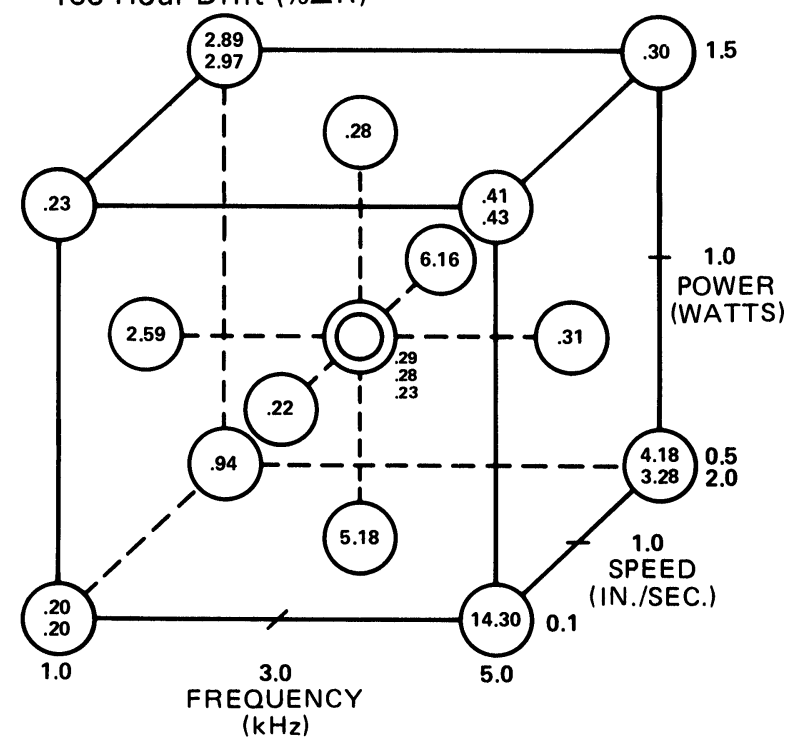

FIGURE 9 Laser trimming of "Birox" $1441(10 \mathrm{k} \Omega / \mathrm{sq}$.) Initial \% deviation from target (precision of trim).

deviation from the target value. The high negative numbers at the left end, back edge of the cube indicate that the material was not trimmed cleanly. This is a case where the speed is too high for the frequency, resulting in a series of unconnected cuts.

Analysis of the cube presenting drift data indicates that optimum trim conditions lie within a diagonal volume running from the bottom left front corner to the top right back corner. The combination of best precision and lowest drift generally is achieved with the lowest speed at the lowest frequency and the lowest power. Drift values generally increase under conditions of high power/high speed/low frequency and low power/high speed/high frequency.

An effort was made to correlate the data with some physical phenomena. Samples trimmed at the various conditions were cross-sectioned and examined using an ETEC scanning electron microscope. 
Initial \% Deviation From Target (Precision of Trim)

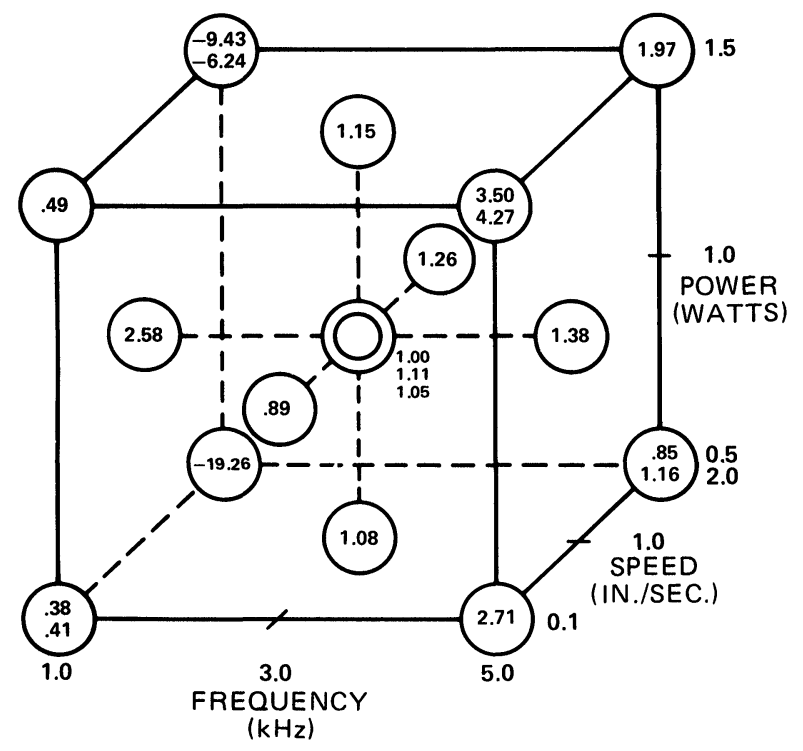

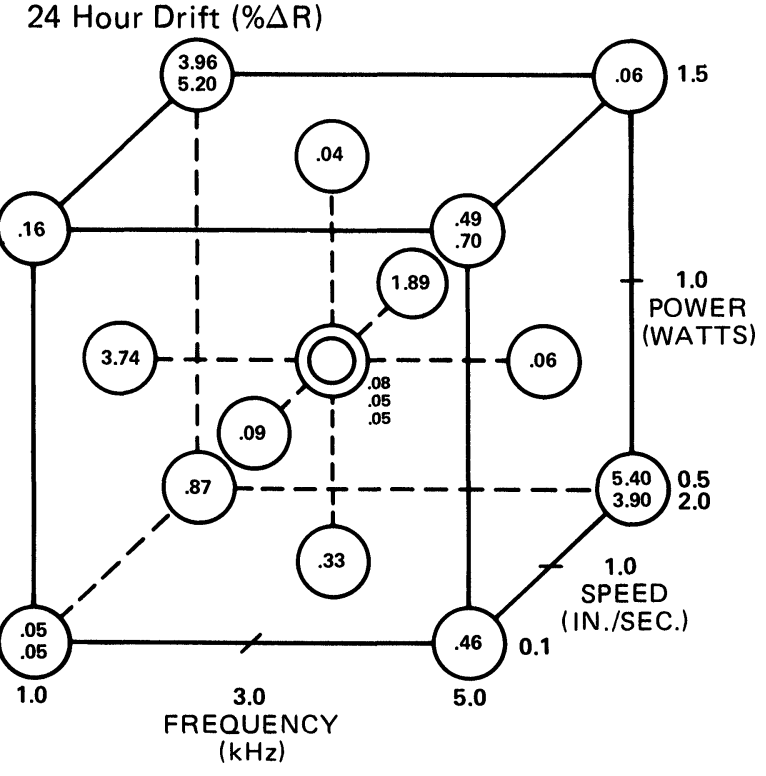

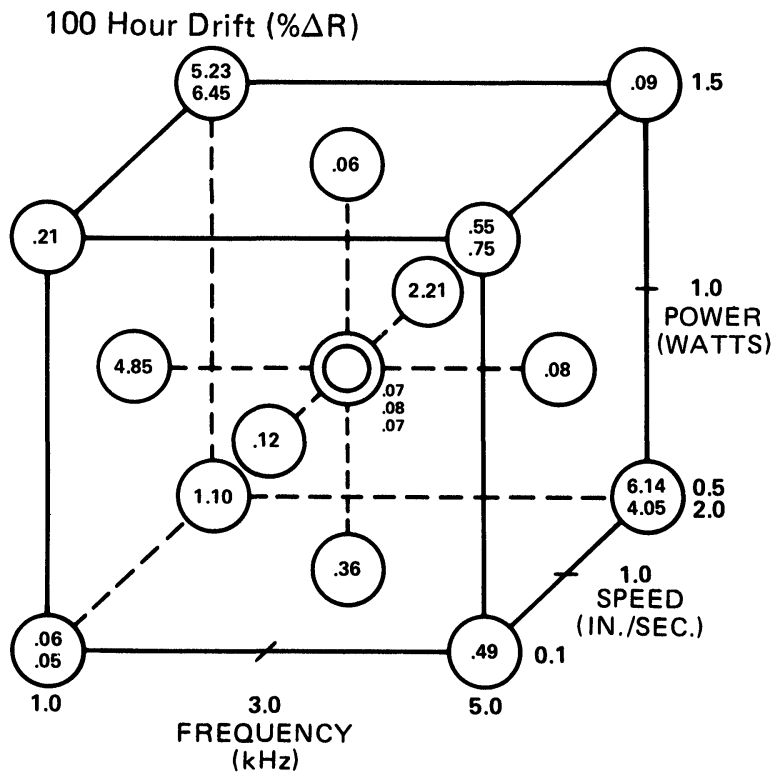

FIGURE 10 Laser trimming of "Birox" 1461 (1 M $\Omega /$ sq.) Initial \% deviation from target (precision of trim).

An interesting observation was made in a comparison of resistors trimmed at the same average power, but at two different frequencies. Figure 11 shows a cross section of a laser kerf cut at $3 \mathrm{kHz}$. Figure 12 shows the same material cut at a $Q$-rate of $5 \mathrm{kHz}$. Penetration of the substrate is obvious in the lower frequency cut. The resistors cut at the higher frequency - no penetration of the substrate - were less stable. Apparently, a kerf must penetrate the substrate
$(>5 \mu \mathrm{m})$ to insure adequate isolation resistance, particularly at high resistivities.

In some instances it was found that resistors trimmed with no penetration of the substrate became unstable when subjected to further elevated temperature hybrid processing. It was found that a quick, production-oriented test which can be run to determine the quality of the laser kerf consists of measuring the insulation resistance (IR) of the kerf 


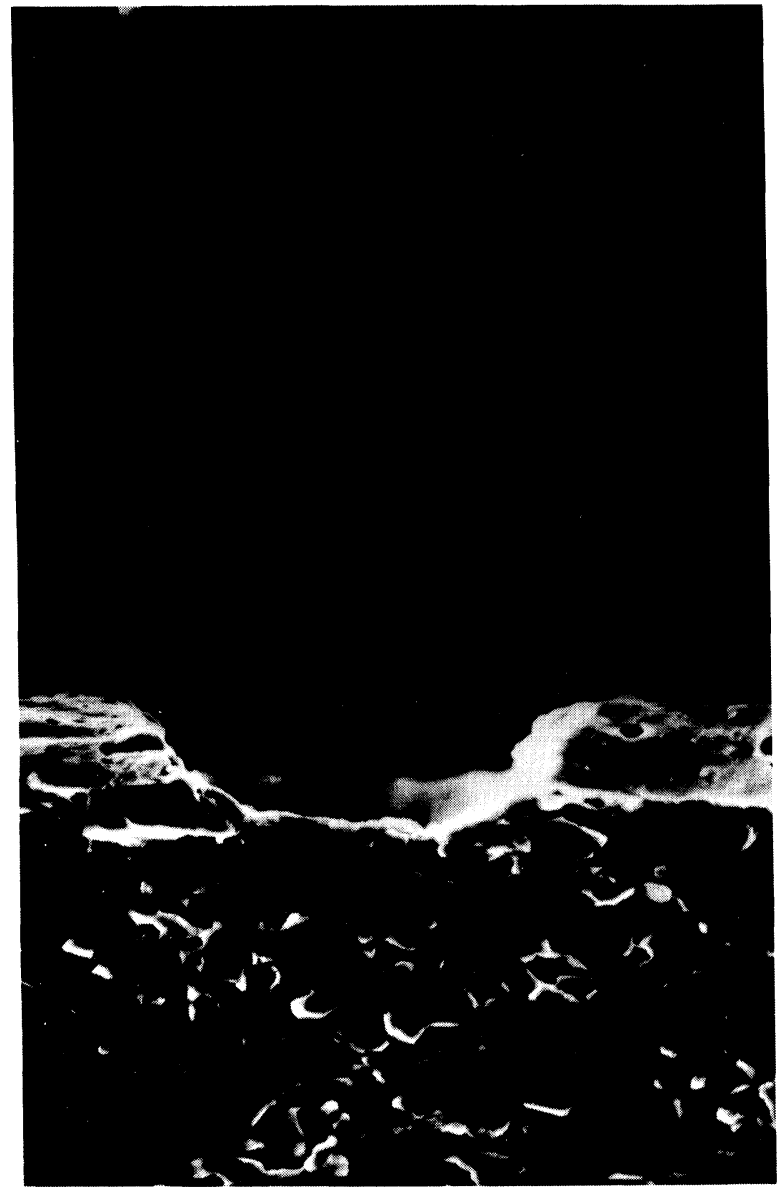

FIGURE 11 Scanning electron micrograph of a thick film resistor laser-trimmed at a frequency of $3 \mathrm{kHz}$. Length of bottom edge : $50 \mu \mathrm{m}$.

of a resistor which has been cut completely through. The resistor is then exposed to $425^{\circ} \mathrm{C}$ for 10 minutes and the IR is remeasured. The IR of a stable resistor will remain unchanged; the IR of an unstable resistor will decrease by one to two orders of magnitude.

The critical nature of kerf isolation with high resistivity films can be understood by thinking of the kerf as a parallel resistor distributed across the trimmed resistor. Consider a 10 megohm resistor with a kerf 100 mil long by 1 mil wide. This kerf is equivalent to a .01 square resistor. A marginally clean kerf of this size could easily vary in resistance over a range of 100 megohm to 10,000 megohm. This is sufficient to produce a $10 \%$ variation in the trimmed 10 megohm resistor. The same range of kerf resistance would cause virtually no effect in a $10 \mathrm{kohm}$, or lower value resistor. A general rule of thumb is that a trimmed resistor requires a total kerf

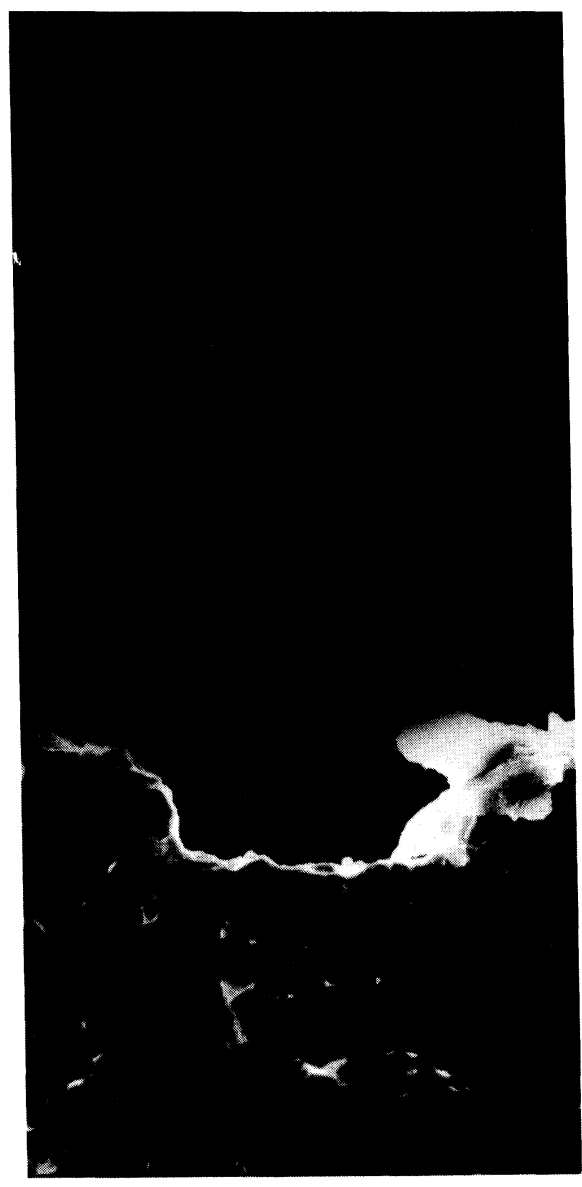

FIGURE 12 Scanning electron micrograph of a thick film resistor laser-trimmed at a frequency of $5 \mathrm{kHz}$. Length of bottom edge : $50 \mu \mathrm{m}$.

resistance not less than $10^{3}$ times the trimmed value to maintain stability to $0.1 \%$, all other factors being excluded.

\section{OTHER FACTORS AFFECTING PRECISION AND STABILITY}

The experiment described in the preceding section was carried out with one type of resistor material. All resistors were trimmed with a single plunge cut which raised the mean value of the resistors by a factor of 1.5. It is possible to improve precision and stability by using multiple plunge cuts or L-cuts and limiting the extent of the cut. Figure 13 illustrates a single plunge cut of a square resistor. If the drift mechanism is defined as the propagation of microcracks from the end of the laser kerf which interrupts 
(A)

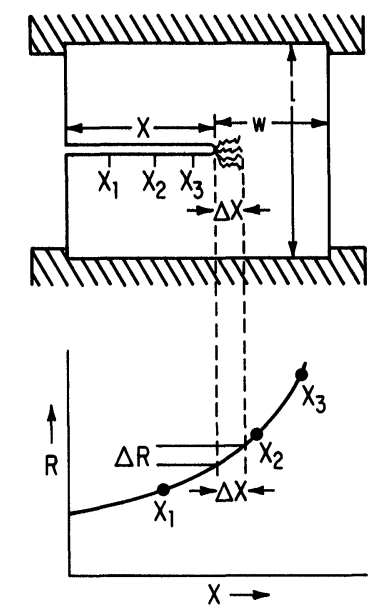

(C)

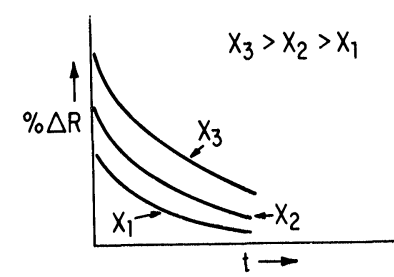

(D)

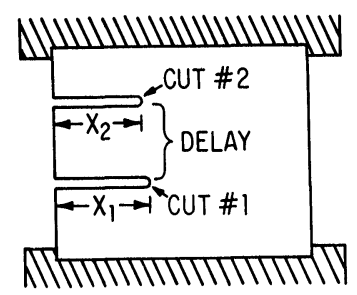

(E)

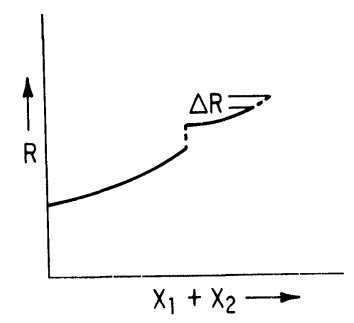

FIGURE 13 Cut mode.

the current flow path and causes an increase in resistance, the extent of propagation $(\Delta X)$ has a greater effect on resistance when the kerf penetrates to depth $X 3$ than to $X 1$.

An attempt was made to correlate the drift observed in the experiment described in the preceding
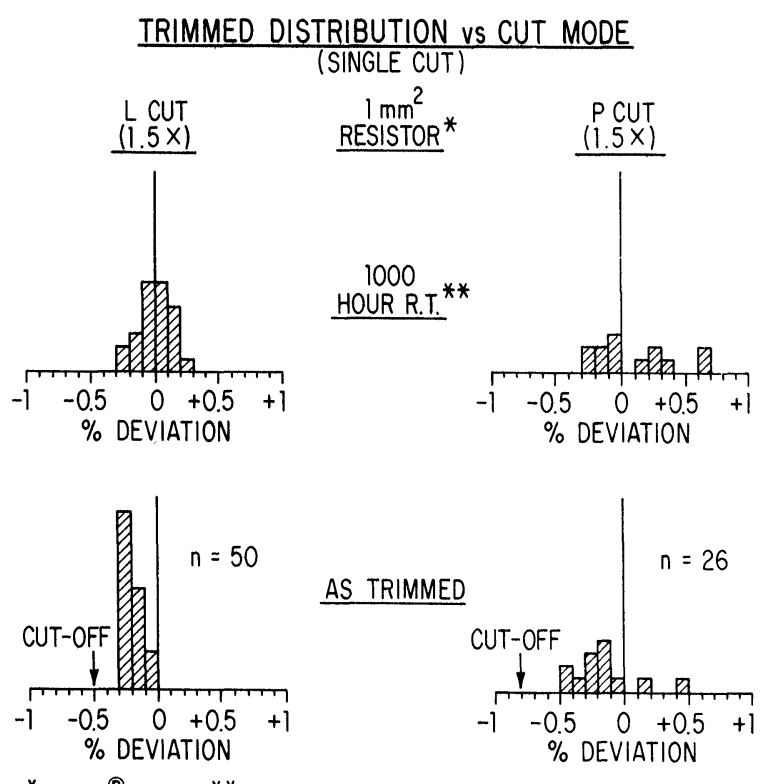

${ }^{*}$ BIROX ${ }^{\circledR} 1651 \quad$ ***oOM TeMPERATURE $23 \pm 1^{\circ} \mathrm{C}$

FIGURE 14 Trimmed distribution vs. cut mode.

section to the extent of cut. Within the constraints of the experiment (maximum $2 X$ trim), no correlation was seen. However, if the end of the cut is placed parallel to the current flow path (via an $L$-cut), then one would expect that precision will be improved and drift reduced. This is indeed true and is shown by the histograms in Figure 14. Another way to improve precision and stability is the use of a double plunge cut with a delay of a few hundred milliseconds between the first and second cut. A method of achieving this in production is to make first cuts in all resistors and then follow with the second cuts.

Figure 15 shows the extent of penetration required to achieve a given resistance change in a square resistor. It is interesting to note that to raise resistance by a factor of two, a $67 \%$ penetration of its width is required.

To limit the extent of cut, a resistor series which has consistent, reproducible and predictable resistivity is desired. The material should be blendable for both resistivity and TCR. Lot-to-lot reproducibility assures that the values will remain close to the design value and, therefore, that the extent of trim will be consistent from month to month. A small coefficient of variation within a fired lot is important to insure that the extent of trim does not vary widely. In our experiments, the total spread of resistance values was $\pm 15 \%$ around a mean. Had the spread been $\pm 30 \%$ (a CV of $10 \%$ ), some samples would have required the resistance to be raised $3 X$; 


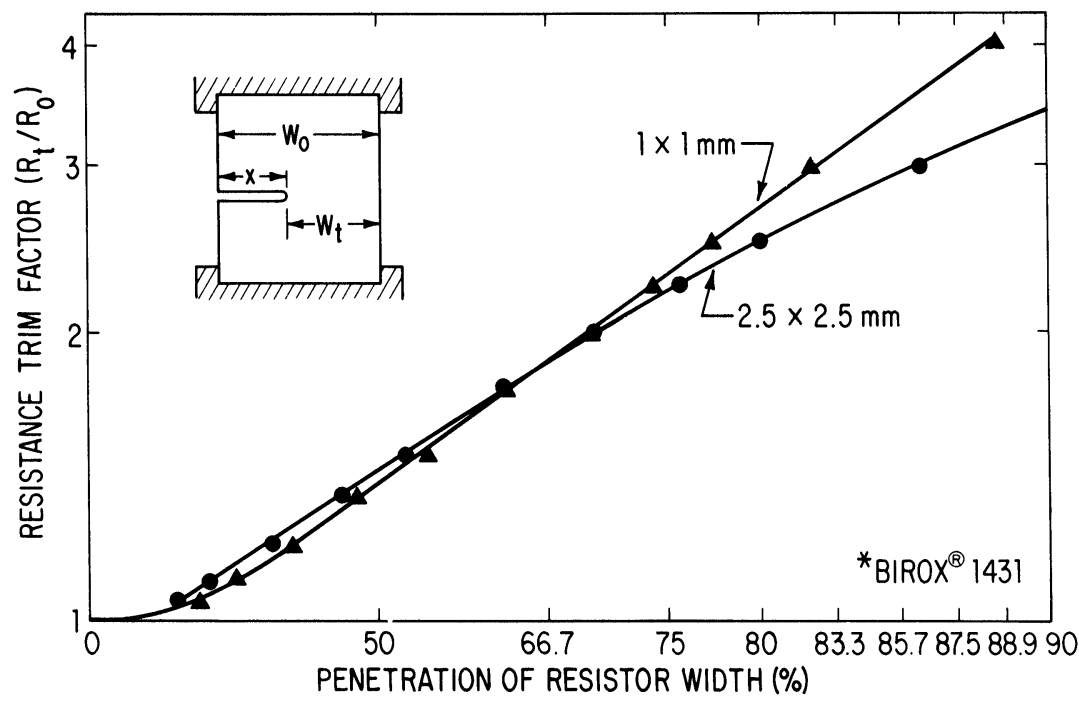

FIGURE 15 Trim characteristic. One square - P Cut.

i.e., the kerf would have to extend through $80 \%$ of the resistor width. Neglecting the effect this has on resistor power handling capability, trimming a resistor to this extent has very dramatic effects on both precision and stability.

The influence of sheet resistivity upon trimmed resistor stability is related to differences in fired print thickness. In general, low resistivity compositions require higher functional phase loading than do high resistivity materials. This results in thicker fired prints. During trimming, the vaporized material is often sufficiently dense, at the point of trim, to attenuate the laser beam intensity and reduce cutting efficiency. (Staining of the kerf floor with condensed material is not uncommon. Although such staining appears to be innocuous, in most cases it does prevent a reliable inspection of kerf quality by visual methods). The stability of a thick film resistor can be improved by reducing its fired thickness. Of course, the increase in sheet resistivity must be accounted for in product design.

\section{CONCLUSIONS}

1. The lowest speed consistent with productivity and precision should be selected.

2. Care should be exercised in selecting the proper average beam power.

3. Decreasing the $Q$-rate can be an effective way of increasing peak power while maintaining a constant average power. This can be used to advantage when trimming lower resistivity materials.

4. The extent of trim should be limited to $2 X$ the fired value.

5. L-cuts or multiple plunge cuts increase the precision and reduce drift.

6. The laser cut should be made with sufficient peak power to penetrate the substrate to a minimum of $5 \mu \mathrm{m}$.

7. An insulation resistance (IR) test with exposure to $425^{\circ} \mathrm{C}$ for 10 minutes can be used to assure the integrity of the kerf.

8. Trim stability can be improved by reducing resistor film thickness. 

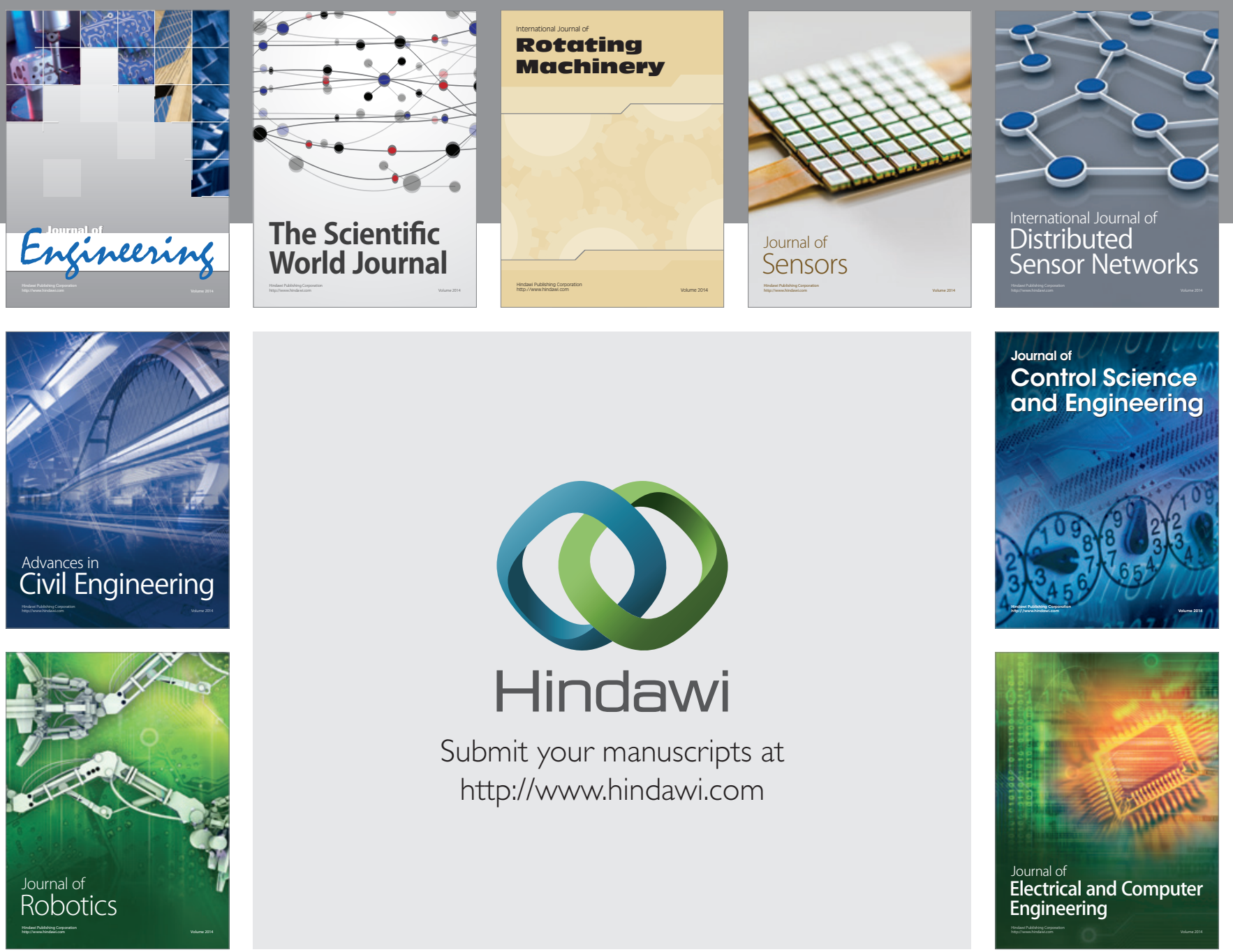

Submit your manuscripts at

http://www.hindawi.com
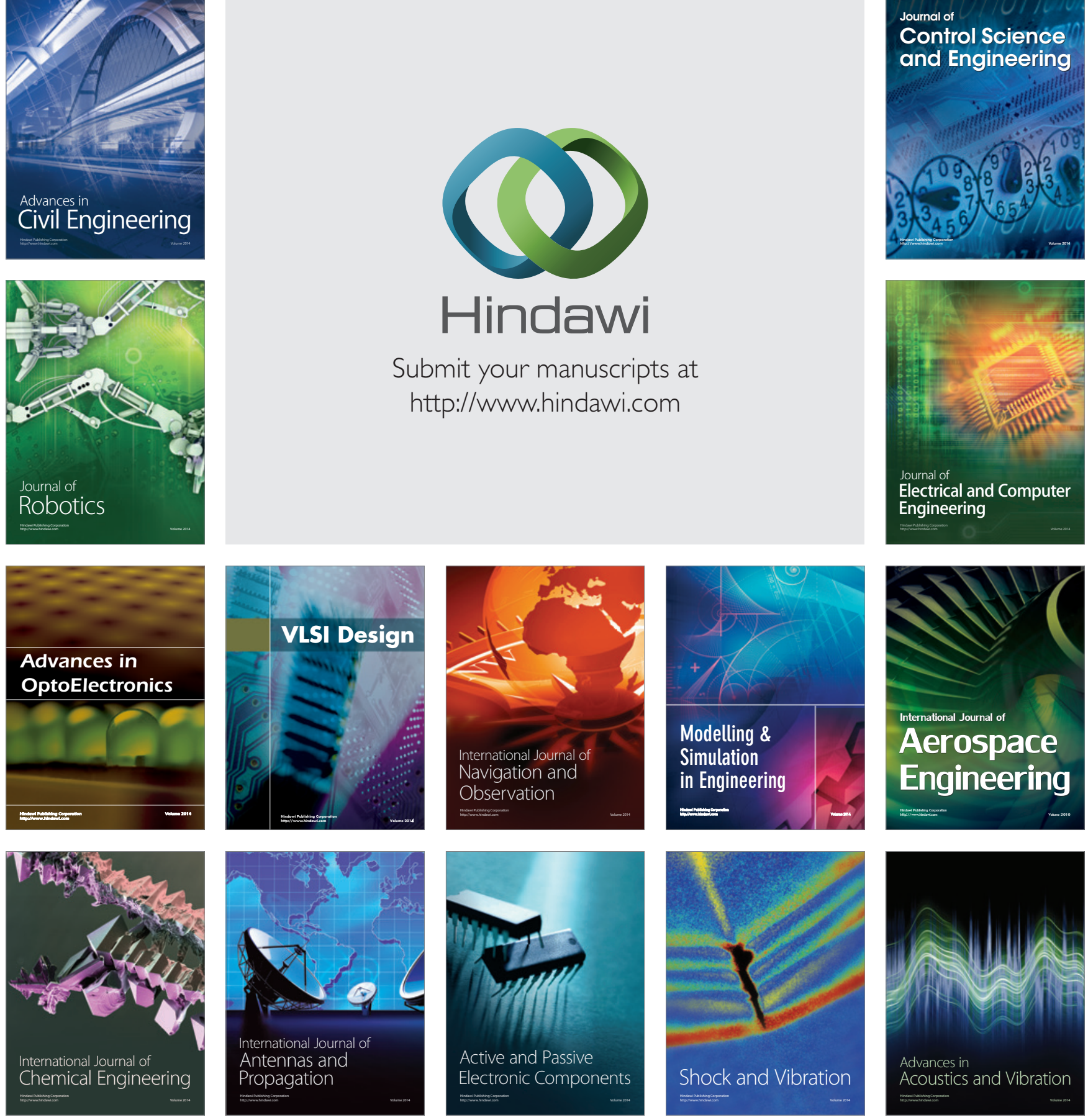